\title{
ANALISIS DEL DESEMPEÑO OCUPACIONAL DE UN NIÑO CON TRASTORNO POR DÉFICIT DE ATENCIÓN E HIPERACTIVIDAD.
}

\section{Analysis Of The Occupational Performance Of A Child With Attention Deficit And Hyperactive Disorder.}

\author{
${ }^{\mathrm{i}}$ Ana Maria Indaburu Espinosa \\ ii Luzia Iara Pfeifer
}

\section{RESUMEN}

Este estudio tuvo como objetivo analizar el desempeño ocupacional de un niño con trastorno por déficit de atención e hiperactividad trastorno por déficit de atención e hiperactividad trastorno por déficit de atención e hiperactividad (TDAH) durante el ejercicio de sus tareas diarias. La metodología escogida fue el estudio de caso de un niño con diagnóstico clínico de TDAH de una ciudad al interior del estado de Sao Paulo, Brasil. La recolección y análisis de datos fue realizada mediante la observación y la aplicación de evaluaciones estandarizadas como Pediatric Evaluation of Disability Inventory (PEDI) traducida y avalada para la población brasilera, School Funtion Assessment (SFA), Questionário sobre o comportamento lúdico infantil (PFEIFER, 2006) y Roteiro de análise de habilidades sociais (ANHÃO, 2008). Estas evaluaciones fueron aplicadas con el fin de abarcar todas las áreas ocupacionales de los niños: juego, participación social, actividades de la vida diaria (AVD) y escolar. Los resultados mostraron que las alteraciones presentadas en el desempeño ocupacional están relacionadas con la calidad en la ejecución de tareas diarias y no con la independencia en la realización de las mismas. Por esta razón no existe ninguna restricción en la participación del niño en los contextos donde se desenvuelve.

Palabras claves: Desempeño ocupacional, Trastorno por déficit de atención e hiperactividad,

\begin{abstract}
The objective of this study was to analyze the occupational performance of the daily activities of children with the ADHD (Attention deficit and hyperactive disorder). The selected methodology was the case study of a boy with a clinical diagnose of ADHD in a city in the interior state of Sao Paulo, Brazil. The recollection and analysis of data were made by observation and the application of standard evaluations like Pediatric Evaluation of Disability Inventory (PEDI), translated and applied in Brazilian population, School function Assessment (SFA), Questionário sobre o comportamento lúdico infantil (PFEIFER,

\footnotetext{
i Investigadora. Estudiante Terapia Ocupacional Universidad del Rosario Bogotá-Colombia e.mail: any_indaburu@yahoo.es

ii Orientadora de la investigación. Professora Doutora do Curso de Terapia Ocupacional da Faculdade de Medicina de Ribeirão Preto - Universidade de São Paulo - MRP - USP. Ribeirão Preto - SP. e-mail: luziara@fmrp.usp.br
} 
2006) and Roteiro de análise de habilidades sociais (ANHÃO, 2008). This evaluation were applied with the objective of include all the occupational areas of children, play, social participation, daily activities and study. The results showed that the alteration presented in the occupational performance is related with the quality in the execution of daily tasks and not with the independence in the accomplishment of them. For this reason, there weren't found any restriction in the participation of the child in his occupational contexts.

Key words: Occupational Performance, Attention deficit and hyperactive disorder

\section{INTRODUCCIÓN}

La intervención de terapia ocupacional en el contexto escolar está divida en las acciones ejercidas en educación regular y educación especial. La mayoría de casos remitidos para terapia ocupacional son niños con dificultades en la atención e inquietud motora siendo el trastorno por déficit de atención e hiperactividad (TDAH) el trastorno neurológico más frecuente dentro de la población infantil ${ }^{1}$.

Los primeros síntomas observados del TDAH son dificultad para concentrarse en una actividad manteniendo atención en detalles, impulsividad, inquietud motora o imposibilidad de permanecer en un lugar por periodos prolongados de tiempo. En cuanto a la participación social, los niños tienen problemas en establecer relaciones interpersonales, labilidad emocional, baja tolerancia a la frustración, baja autoestima y comportamientos desafiantes ${ }^{2}$.

Según el DSM-IV-TR algunos síntomas hiperactivo-impulsivos deben estar presentes antes de los siete años de edad pero muchos niños son diagnosticados tardíamente a pesar de presentar previamente los síntomas.

Una característica importante para el diagnóstico del TDAH es la necesidad de que los síntomas estén presentes por lo menos en dos contextos en los que el niño se desenvuelve, por ejemplo, la casa y el colegio ${ }^{\text {(Facion, 1991) }}$. Además, deben existir claras evidencias de interferencia en el desempeño ocupacional social o académico del mismo ${ }^{3}$.

Según la AOTA ${ }^{4}$, los terapeutas ocupacionales tienen como objetivo ayudar a las personas a desarrollar actividades diarias que sean significativas para ellas direccionando la intervención en sentido de auxiliar su desempeño en las diferentes áreas de ocupación: actividades de la vida diaria (AVD), actividades instrumentales de la vida diaria (AIVD), educación, trabajo, juego, ocio productivo y participación social.

Debido a lo descrito anteriormente, la finalidad de este estudio fue analizar el desempeño ocupacional de un niño escolarizado con TDAH durante el ejercicio de sus tareas diarias. 


\section{METODO}

En esta investigación se utilizó la metodología de estudio de caso que pretende, basado en antecedentes teóricos, y mediante varias fuentes de recolección de datos, dar respuesta a la pregunta de investigación a través del análisis de un único participante ${ }^{5}$.

El tipo de estudio es descriptivo y el método de recolección y análisis de datos es cuali-cuantitativa. Fueron utilizadas tanto evaluaciones estandarizadas como observación del desempeño en la casa y el colegio del niño.

\section{$\underline{\text { Participante }}$}

Las características de inclusión del participante fueron: edad, escolarización y diagnóstico clínico de TDAH. Fue seleccionado un participante de sexo masculino de 10 años procedente de una ciudad del interior del estado de Sao Paulo, Brasil, el cual se encuentra escolarizado y actualmente cursa tercera serie en la jornada de la tarde. La familia se encuentra conformada por sus padres y dos hermanos, uno mayor de 12 años y uno menor de 5. La familia del participante pertenece al estrato socio-económico bajo y cuentan con el ingreso únicamente del padre.

La familia firmó el correspondiente consentimiento informado, autorizando el estudio y publicación del caso.

Tanto este proyecto como el consentimiento informado fueron analizados por el comité de ética en investigación y aprobado el 26 de Mayo de 2008 de acuerdo con el proceso HCRP n 4104/2008.

\section{$\underline{\text { Instrumentos }}$}

Las evaluaciones aplicadas fueron escogidas con el fin de abarcar las principales áreas ocupacionales de los niños.

Los instrumentos seleccionados fueron: Pediatric Evaluation of Disability Inventory (PEDI) (Inventario pediátrico de evaluación de la discapacidad) traducida y avalada para la población brasilera ${ }^{6}$, School Funtion Assessment (SFA) (Evaluación del funcionamiento escolar) ${ }^{7}$ Questionário sobre o Comportamento Lúdico Infantil (Cuestionario sobre el comportamiento lúdico infantil) ${ }^{8}$ y Roteiro de Análise de Habilidades Sociais (guía de análisis de habilidades sociales) ${ }^{9}$.

El PEDI es una evaluación que tiene como objetivo proveer información acerca del desempeño funcional del niño y el nivel de asistencia del cuidador en áreas de autocuidado, movilidad y funciones sociales, las cuales fueron evaluadas en el presente estudio. Esta evaluación, ha sido usado en la intervención clínica y

en diversas investigaciones científicas ${ }^{10} 11{ }^{12}$ y fue normativizado para la población brasilera en el 2005 (Manzini, 2005).

El objetivo del SFA es obtener información acerca del desempeño funcional del niño en las actividades desarrolladas en el colegio (Coster 1998). Esta evaluación contiene 3 ítems: participación en los principales ambientes escolares, auxilio en el desempeño de las tareas y el desempeño en las actividades. El SFA fue utilizado por Silva ${ }^{13}$ y mostró que esta evaluación es un instrumento adecuado en la 
identificación de alteraciones del desempeño escolar, señalando aspectos que pueden ser adaptados en el ambiente escolar para mejorar la participación de los niños con alteraciones neuropsicomotoras.

El Questionario sobre o comportamento lúdico infantil (PFEIFER, 2006) brinda información acerca de las preferencias en juegos, actividades deportivas y artísticas y de la rutina del niño. Fue aplicada con el propósito de abarcar el área ocupacional de juego. Este cuestionario está compuesto por 19 ítems, de los cuales los primeros 8 estas relacionados a la caracterización del niño. Luego brida información acerca de con quien juega el niño, donde juega el niño, a que juega, que ve en la televisión y cuantas horas diarias ve televisión. También indaga sobre si el niño practica alguna actividad deportiva, cultural o artística, si suele ir a eventos culturales, que actividades practica con sus padres, que suele leer y finalmente su rutina diaria y semanal ${ }^{\text {(PFEIFER, 2006) }}$. Fue utilizado por Terra e Pfeifer ${ }^{14}$ y aplicado a 32 niños, siendo 16 de ellos diagnosticados con TDAH y 16 sin alteraciones en el desarrollo neuropsicomotor. La conclusión de dicho estudio, mostró que es un instrumento adecuado para comprender las diferencias y semejanzas en el juego de esos niños (TERRA, PFEIFER, 2009).

Finalmente, el Roteiro de análise de habilidades sociais desarrollado por Anhão (ANHAO, 2008), permite analizar el área de participación social durante la interacción del niño con pares. El instrumento propone la evaluación de 15 comportamiento evaluados durante la interacción del niño como se presenta a continuación: hay interacción con otro niño, hay interacción con la educadora, hay interacción con objetos, disputa la atención de la profesora con otra persona, hay peleas o agresiones, tiene autodefensa, establece contacto inicial con otra persona, juega con diferentes objetos, juega con un solo tipo de objeto, llora, sonríe, permanece solo, canta, imita otros niños y imita a la profesora (ANHAO, 2008) . Este instrumento fue utilizado por Anhão, Pfeifer y Santos ${ }^{15}$, el cual después de la aplicación del test Alpha de Cronback fue observada la confiabilidad inter-examinadores demostrando que el mismo es adecuado para captar el desempeño social de los niños y puede ser reproducido en otras investigaciones.

\section{$\underline{\text { Procedimiento para colecta de datos }}$}

Los datos fueron obtenidos mediante la aplicación de los instrumentos estandarizados, observación y registro de la actividad lúdica del participante.

El PEDI es aplicado al cuidador principal siendo en este caso la mamá del participante la encargada de dar las informaciones requeridas. La entrevista previa y aplicación de la evaluación fue realizada en la casa del participante, siguiendo cada parte propuesta por el instrumento. Las respuestas fueron registradas con el fin de analizar posteriormente detalles significativos dados por la cuidadora.

El SFA fue aplicado a la profesora la cual dio su punto de vista acerca del participante respecto a sus compañeros de sala en las tres áreas evaluadas: participación, asistencia y desempeño. La evaluación fue aplicada en la sala de profesores, con el fin de evitar interrupciones e interferencias durante la entrevista.

“Questionário sobre o comportamento lúdico infantil” (PFEIFER, 2006) es aplicado al niño, el cual contesta preguntas acerca de sus preferencias lúdicas. El primer contacto con el participante y su familia y la aplicación del cuestionario fue realizado durante una consulta en el ambulatorio de Neurodesarrollo infantil en un hospital de tercer nivel en el interior de Sao Paulo. 
El "Roteiro de análise de habilidades sociais" (ANHAO 2008) permite analizar las conductas de interacción social durante una filmación de 15 minutos. El niño fue filmado durante el periodo de descanso de su jornada escolar, mientras participaba de un partido de fútbol con sus compañeros.

Las observaciones fueron realizadas en la casa del niño con el fin de tener una mejor perspectiva de la ejecución en las AVD. En el colegio se observó el comportamiento intraula y se registró el desempeño en el juego e interacción social durante el descanso.

\section{Procedimiento para análisis de datos:}

Los datos obtenidos fueron analizados según lo propuesto por cada evaluación.

Para el PEDI fue utilizada la puntuación estándar con la finalidad de analizar cuáles de los ítems evaluados están dentro del parámetro esperado y cuáles se salen del rango. Se tuvieron en cuenta los tres aspectos evaluados (cuidados personales, movilidad y función social) y serán presentados únicamente los datos relevantes para la investigación que corresponden a los ítems que se salen del rango y que serían los objetivos de intervención.

El SFA fue creado bajo la teoría Rasch, que maneja escalas teniendo en cuenta la complejidad de cada ítem. Por esto, en el SFA, fue puntuado cada uno de los ítems de forma general teniendo en cuenta el score bruto y el score de criterio. Ya que el objetivo de la evaluación es analizar los aspectos que interfieren en el desempeño, los scores de corte no se tuvieron en cuenta. Se dio énfasis solamente a las tareas de cada ítem que puntuaron menos de setenta y cinco (75) en el score de criterio.

El Questionário sobre o comportamento lúdico infantil (PFEIFER, 2006) y el Roteiro de análisis de habilidades sociais (ANHAO 2008) fueron analizados cualitativamente por el evaluador teniendo en cuenta las respuestas del participante y el video registrado.

\section{RESULTADOS}

A continuación serán presentados los resultados obtenidos durante el proceso, relacionando los resultados cualitativos de evaluaciones estandarizadas con las observaciones realizadas.

\section{$\underline{\text { PEDI }}$}

Respecto a las habilidades funcionales evaluadas, no se evidencia ninguna dificultad en la independencia de las AVD. El puntaje obtenido fue 100\% de los ítems. Sin embargo, en la observación que se llevo a cabo en la casa durante la realización de tareas diarias como bañarse, vestirse, almorzar y alistar los materiales para el colegio se identificaron fallas en la calidad del desempeño de las tareas debido al olvido o desatención en la ejecución y finalización de las mismas. Fue necesaria la intervención de la cuidadora, la cual retroalimentaba sobre los detalles olvidados por el niño.

En el ítem de movilidad no fue referido ningún problema en la ejecución de las actividades consideradas dentro del mismo. El puntaje obtenido en el PEDI fue del 100\% 
Respecto a las funciones sociales, de los 65 ítems evaluados, el participante obtuvo 59 lo que significa un 90,7\%. Los ítems no alcanzados por el niño fueron los que se refieren a las dificultades para relacionar eventos ocurridos en el pasado y así lograr contar una historia con lógica temporal. También se identificaron problemas en la interacción en juegos grupales debido a la dificultad en seguir reglas y baja tolerancia a la frustración. Respecto al ítem de auto-información, no fue puntuado el que tiene que ver con pedir y dar datos acerca de ubicación espacial cuando es requerido desplazarse de un lugar a otro. En cuanto a la orientación espacial no consigue identificar las horas en un reloj ni regular el tiempo necesario para la ejecución de tareas. En las tareas domésticas los están ligados la dificultad en terminar con una instrucción dada debido a la falta de atención en los pasos y a la distractibilidad con detalles no relevantes

En cuanto a la segunda parte de la prueba relacionada con la ayuda del cuidador, la mayor asistencia requerida tiene que ver con la necesidad de reforzar o recordar detalles que son olvidados por el niño en el momento de reproducir pasos indispensables para ejecutar tareas. Además, las actividades que requieren de movimientos finos o precisos son auxiliadas por la cuidadora, ya que la inquietud motora e impaciencia no le permiten realizarlo de manera adecuada.

Durante la observación del desempeño que se llevo a cabo en la casa mientras el niño ejecutaba tareas cotidianas, fue posible comprobar las dificultades descritas durante la entrevista con la cuidadora en cuanto a la calidad de la realización de las tareas y seguimientos instruccional.

$\underline{\text { SFA }}$

Fue realizada la entrevista con la profesora principal del niño, la cual no relató ningún problema académico relacionado con contenidos.

En el ítem de participación no se identificó ninguna barrera en los ambientes evaluados (Tabla 1)

Tabla 1: Resultado de evaluación de participación en ambiente escolar

\begin{tabular}{|l|c|c|}
\hline & SCORE BRUTO & SCORE CRITERIO \\
\hline PARTICIPACION & 36 & 100 \\
\hline
\end{tabular}

En cuanto a la segunda parte evaluada perteneciente al Auxilio en las tareas, los resultados muestran que el niño requiere mayor asistencia (Asistencia moderada) por parte de la profesora en tareas físicas en el ítem relacionado con la organización de materiales. Los ítems puntuados con asistencia mínima fueron: permanencia en sedente durante actividades escolares y manejo de materiales, debido a la dificultad en la pérdida de los mismos por descuido del niño (Tabla 2).

Respecto a las tareas cognitivo/comportamentales, los ítems que están más alejados del score criterio requiriendo asistencia moderada son la finalización de tareas y el control del comportamiento durante las actividades realizadas en aula. (Tabla 2). 
Para ninguna de las dos tareas son requeridas adaptaciones.

Tabla 2: resultados de evaluación de Asistencia en tareas

\begin{tabular}{|l|c|c|}
\hline & SCORE BRUTO & SCORE CRITÉRIO \\
\hline Tareas Físicas & 31 & 70 \\
\hline Tareas Cognitivas & 30 & 66 \\
\hline
\end{tabular}

Respecto al tercer ítem relacionado con el desempeño, las mayores dificultades identificadas tienen que ver con las tareas cognitivas donde se obtuvieron los puntajes más bajos. (Tabla 3)

Tabla 3: Resultado de desempeño en tareas físicas e cognitivas

\begin{tabular}{|c|c|c|}
\hline & SCORE BRUTO & SCORE CRITÉRIO \\
\hline \multicolumn{3}{|l|}{ TAREAS FÍSICAS } \\
\hline Desplazamiento & 75 & 95 \\
\hline Mantenimiento y cambio de posición & 46 & 83 \\
\hline Actividades recreativas & 44 & 100 \\
\hline Manipulación con movimiento & 60 & 78 \\
\hline Utilización de materiais & 100 & 100 \\
\hline Organización y limpieza & 81 & 83 \\
\hline Comer y beber & 53 & 78 \\
\hline Higiene & 60 & 100 \\
\hline Manipulación de ropa & 68 & 100 \\
\hline Subir y bajar escaleras & 24 & 100 \\
\hline Trabajos escritos & 45 & 83 \\
\hline Uso de computadores & 32 & 100 \\
\hline \multicolumn{3}{|l|}{ TAREAS COGNITIVAS } \\
\hline Comunicación funcional & 52 & 100 \\
\hline Memoria y comprensión & 38 & 85 \\
\hline Seguridad & 40 & 100 \\
\hline Auto cuidado & 40 & 100 \\
\hline Interacción Positiva & 72 & 76 \\
\hline Control del Comportamiento & 43 & 68 \\
\hline Seguimiento de reglas/Expectativas sociales & 45 & 77 \\
\hline Obediencia a ordenes de adultos y reglas & 58 & 86 \\
\hline Comportamiento/conclusión de tareas & 54 & 56 \\
\hline
\end{tabular}


El ítem que obtuvo la menor puntuación fue conclusión de las tareas. Según el score criterio, las acciones de este ítem que están por debajo del puntaje obtenido y que según el SFA tienen desempeño parcial son las relacionadas con mantener la atención en una tarea por quince minutos, iniciación de actividades después de dada la instrucción, modificación del desempeño después de dada una retroalimentación y hace adecuado uso del tiempo dado para cada actividad. Dentro de las tareas puntuadas con desempeño inconsistente están la resolución autónoma de problemas y permanencia por más de veinte minutos en el puesto prestando atención.

Otras dificultades presentadas por él niño tienen que ver con el ítem de control del comportamiento, especialmente a lo que se refiere a tolerancia a la frustración, aceptación de críticas y reacciones agresivas puntuadas también como desempeño parcial.

Respecto al desempeño en AVD realizadas dentro del horario escolar, como entrar al baño y comer, no se presentaron dificultades relatadas durante la entrevista (desempeño consistente).

Otros problemas identificados pero menos relevantes estadísticamente tienen que ver con la relación entre pares y seguimiento de reglas durante actividades grupales los cuales fueron puntuados con desempeño inconsistente.

Durante la observación intraula se observaron episodios de desatención y distracción con objetos no relevantes para la actividad. La sala de aula no tiene elementos distractores en paredes que puedan favorecer las dificultades del niño. La ubicación dentro del salón, impide el control y la adecuada supervisión por parte del profesor y favorece la desatención con otros compañeros.

\section{Questionário sobre o comportamento lúdico infantil}

Este cuestionario aplicado al niño muestra las preferencias lúdicas, artísticas y deportivas. En cuanto a las personas con las que interactúa a la hora de jugar, el niño prefiere jugar con los compañeros del colegio, hermanos, vecinos, niños del mismo sexo y los papás.

No se evidencia preferencia específica por algún lugar para jugar. Sus juegos favoritos son: carritos, fútbol, escondidas, elevar cometa, hacer colecciones, juegos de construcción, video game, juegos de computador, pintar y dibujar.

Ve una hora de televisión diaria prefiriendo los dibujos animados. Realiza actividad artística de pintura y música tres veces a la semana. Frecuentemente visita a los abuelos, otros familiares y asiste a actividades religiosas. Le gusta leer historietas.

Su rutina diaria está dada por clases de pintura y música por las mañanas y colegio por la tarde. Los fines de semana asiste a la iglesia y juega.

Fue posible evidenciar la interacción con los vecinos durante un juego de fútbol en el barrio. Además, se constató que los videos juegos y juegos de computador son actividades que realiza sólo. Impone sus reglas y se le dificulta escuchar a los demás. 


\section{$\underline{\text { Roteiro de análise de habilidades sociais }}$}

La filmación fue realizada durante el período de descanso del colegio. El niño se encontraba jugando fútbol con los compañeros. Durante los primeros siete minutos realizó adecuado contacto con los compañeros interactuando con ellos y el balón. Al minuto ocho se presentó un problema entre el participante y otro niño que requirió la intervención de la profesora. La causa del problema fue la baja tolerancia a la frustración e imposición de reglas por parte del participante. Al minuto diez el juego fue restablecido sin más problemas.

\section{DISCUSIÓN}

Se puede concluir después de la realización del estudio, que las principales causas que pueden afectar el óptimo desempeño ocupacional del niño con diagnostico de TDAH del presente estudio son la incapacidad de fijar la atención en una tarea específica lo que lo lleva a distraerse con otras cosas no relevantes y olvidar su objetivo principal afectando la finalización de la misma. Esto ocurre en la ejecución de tareas escolares y en las AVD. Así mismo, fueron evidenciadas dificultades en el área de participación social, principalmente por la dificultad en el seguimiento de reglas y tolerancia a la frustración.

Según el estudio realizado por Alessandri ${ }^{16}$ los niños con TDAH son menos competentes que sus iguales en actividades grupales y académicas. En contraste, los resultados del estudio mostraron que, aunque las habilidades cognitivas y sociales del participante estén comprometidas, sus relaciones con pares y actividades grupales no se ven afectadas. La mayor dificultad en cuanto a competitividad se evidenció en aula no por su capacidad cognitiva, sino por el hecho de no culminar las actividades propuestas.

Respecto al área de juego y participación social, los principales problemas están relacionados con la impulsividad e imposibilidad de seguir reglas queriendo siempre imponer las de él. Esta conclusión refuerza los resultados obtenidos durante la investigación de Leypod y Bundy ${ }^{17}$ en la cual refieren que los niños con TDAH tienen dificultades en mantener relaciones con iguales. Así mismo, relatan que entre el 50 y $60 \%$ de estos niños experimentan rechazo por parte de sus compañeros.

Según la CIF, existe una restricción en la participación cuando hay problemas que un individuo puede enfrentar durante situaciones de su vida. Así mismo, hay limitación en la actividad cuando existen interferencias durante la ejecución de las actividades ${ }^{18}$.

En la investigación realizada por Hynd ${ }^{19}$ se estudio el desempeño académico de niños con TDAH de diferentes tipos. Este estudio concluyó no hay diferencia en los coeficiente intelectuales de los niños con TDAH pero que el TDAH de tipo desatento tiene peor desempeño en áreas como matemáticas y lectura. Este estudio apoya la conclusión obtenida durante el transcurso de esta investigación la cual apunta como principal causa de problemas en el desempeño ocupacional del participante de este estudio, la falta de atención durante la ejecución de tareas. 
El principal aporte de este estudio de caso fue mostrar que el TDAH no interfiere en la participación, ni ejecución de tareas cotidianas del niño, lo que le permite desempeñarse en todos los contextos ocupacionales sin mayores inconvenientes. En consecuencia, el TDAH afecta el desempeño ocupacional en cuanto a la calidad de las tareas realizadas más no en la ejecución independiente de las mismas.

Las principales limitaciones para la realización de este estudio están relacionadas con el corto tiempo para la recolección de datos y la limitación de la metodología utilizada (estudio de caso) para generar una conclusión más amplia. Así mismo, en las evaluaciones aplicadas no se encontraron dificultades representativas en la independencia del desempeño ocupacional del participante. Debido a esto cabe resaltar la importancia que tuvo la observación del desempeño en los diferentes contextos dentro de este estudio.

Para futuras investigaciones se recomienda limitar las variables a analizar escogiendo solo un área ocupacional con el fin de profundizar el análisis de la misma.

\section{Agradececimientos}

Especial agradecimiento al equipo de infancia de Terapia Ocupacional del Hospital das Clínicas en Ribeirão Preto- SP, por su apoyo y aportes.

A Lauana Nogueira Terra, estudiante de terapia ocupacional de La Universidad de São Paulo por su colaboración en la búsqueda y elección del caso de esta investigación.

\section{REFERENCIAS BIBLIOGRAFICAS}

${ }^{1}$ CELA J, BURSELA E. Revista de Psiquiatría y Psicología del Niño y del Adolescente, 7(1): 110124. 2007

${ }^{2}$ FACION, J.R. Síndrome de Hipercinese: Uma revisão bibliográfica. In: Psicologia Argumento,Ano IV, No. X, PUC Paraná, p. 07-24. 1991

3 GREENHILL LL. Diagnosing attention-deficit/hyperactivity disorder in children. J Clin Psychiatry, 59 (suppl 7):31-41,1998.

${ }^{4}$ AMERICAN OCCUPATIONAL THERAPY ASSOCIATION (AOTA). Occupational therapy practice framework: domain and process. American Journal of Occupational Therapy, v. 56, p. 609 639, 2002.

${ }^{5}$ YIN, R. K. Estudo de caso planejamento e métodos. Porto Alegre : Bookman, 2005.

${ }^{6}$ MANCINI, M. C. Inventário de Avaliação Pediátrica de Incapacidade (PEDI): manual da versão brasileira adaptada. Belo Horizonte: Editora UFMG,208 p. 2005. 
${ }^{7}$ COSTER, W.J. et al. School Function Assesment: user manual. San Antonio, Texas: Therapy Skill Builders, 1998.

${ }^{8}$ PFEIFER, L. I. O brincar de crianças escolares de 6 a 12 anos, diferenças de gênero e geração. Ribeirão Preto. Faculdade de Medicina de Ribeirão Preto, 2006. Relatório final de pesquisa através da prática dos graduandos em terapia ocupacional da disciplina AARTLL.

9 ANHÃO, P. P. G. O processo de interação social na inclusão escolar de crianças com síndrome de Down em educação infantil. Projeto de Pesquisa (qualificação) Programa de pós graduação em saúde comunitária. FMRP - USP, Ribeirão Preto, 2008.

${ }^{10}$ CUSTERS et al. Cultural differences in functional status measurement: analyses of person fit according to the Rasch model. Quality of Life research, v. 9, n. 5, p. 571-578, 2000. ${ }^{10}$ ANHÃO, P. P. G. O processo de interação social na inclusão escolar de crianças com síndrome de Down em educação infantil. Projeto de Pesquisa (qualificação) Programa de pós graduação em saúde comunitária. FMRP - USP, Ribeirão Preto, 2008.

${ }^{10}$ CUSTERS et al. Cultural differences in functional status measurement: analyses of person fit according to the Rasch model. Quality of Life research, v. 9, n. 5, p. 571. 2001

11 KETELAAR et al. Effects of a functional therapy program on motor abilities of children with cerebral palsy. Physical Therapy, v. 81, n.9, p.1534-1545, 2001.

12 MANCINI, M.C. et al. O impacto da asma infantil no perfil funcional de crianças entre um e quatro anos de idade. Revista Paulista de Pediatria, v. 20, n.2, p. 69-77, 2002 b.

13 SILVA, D. B. R. Avaliação das atividades de crianças com paralisia cerebral na escola regular: participação, níveis de auxílio e desempenho. (dissertação). Programa de pós graduação em Educação Especial (Educação do Indivíduo Especial). Universidade Federal de São Carlos, UFSCAR, São Carlos, 2007.

${ }^{14}$ TERRA, L. N.; PFEIFER, L. I. Análise sobre a diferença do brincar entre crianças sem alterações no desenvolvimento neuropsico-motor e crianças com TDA/H - transtorno do déficit de atenção com hiperatividade. Relatório de Pesquisa. FMRP - USP, Ribeirão Preto, 2009.

15 ANHÃO, P. P. G.; PFEIFER, L. I.; SANTOS, J. L. O Processo de Interação Social na Inclusão Escolar de Crianças com Síndrome de Down em Educação Infantil. Revista brasileira de educação especial. Submetido. 2009.

16 ALESSANDRI, S., Attention, play and social behavior in ADHD preschoolers. Journal of Abnormal Child Psychology, 1992. 20(3): p. 289-302.

17 LEIPOLD, E.E.; BUNDY, A.C.. Playfulness in Children with AttentionDeficit Hyperactivity Disorder. The Occupational Therapy Journal of Research, 2000. 20(1): p. 61-79. 
${ }^{18}$ AYUSO-MATEOS JL, NIETO-MORENO M, SÁNCHEZ-MORENOJ, VÁZQUEZ-BARQUERO JL. Clasificación Internacional del Funcionamiento, la Discapacidad y la Salud (CIF): aplicabilidad y utilidad en la práctica clínica. Med Clín (Barc). 2006; 126: 461-6.

19 HYND, G.W.; LORYS, A.R.; SEMRUD-CLICKEMAN, M. et al. - Attention deficit disorder without hyperactivity: A distinct behavioral and neurocognitive syndrome. J Child Neurol 6S:S36-S43, 1991. 